\title{
Condition féminine, rapports sociaux de sexe, genre...
}

Feminine condition, the social relations of the sexes, gender...

\section{Michelle Zancarini-Fournel}

\section{(2) OpenEdition}

12 Journals

Édition électronique

URL : https://journals.openedition.org/clio/9851

DOI : 10.4000/clio.9851

ISSN : 1777-5299

Éditeur

Belin

Édition imprimée

Date de publication : 31 décembre 2010

Pagination : 119-129

ISBN : 978-2-8107-0098-1

ISSN : 1252-7017

Référence électronique

Michelle Zancarini-Fournel, "Condition féminine, rapports sociaux de sexe, genre... », Clio. Femmes, Genre, Histoire [En ligne], 32 | 2010, mis en ligne le 31 décembre 2012, consulté le 29 avril 2022. URL : http://journals.openedition.org/clio/9851 ; DOI : https://doi.org/10.4000/clio.9851 


\section{Regards complémentaires}

\section{Condition féminine, rapports sociaux de sexe, genre...}

Michelle ZANCARINI-FOURNEL

Cet article a pour origine certaines interrogations d'étudiant-e-s du cours de l'IEP de Lyon « Genre et Rapports sociaux de sexe et genre » sur la généalogie de ces concepts et leurs présupposés théoriques respectifs. Mon intention est de reprendre ici l'histoire récente de l'usage des catégories de sexe, de la condition féminine au genre (gender), en passant par les rapports sociaux de sexe, et de prolonger ainsi en l'historicisant le numéro de décembre 1985 du BIEF (Bulletin d'Information des études féminines) ${ }^{1}$, en particulier l'article d'Anne-Marie Daune-Richard et d'Anne-Marie Devreux intitulé «La construction sociale des catégories de sexe $»^{2}$. De nombreuses études ont depuis été consacrées aux catégories de genre ou de rapports sociaux de $\operatorname{sexe}^{3}$; la confection de dictionnaires et de répertoires a permis d'en faire connaitre les définitions plus ou moins développées ${ }^{4}$. Mais je souhaite tenter ici une autre démarche: repérer leur emploi, leur parcours dans le second $\mathrm{XX}^{\mathrm{e}}$ siècle. Pour l'expliciter, je vais prendre un exemple: la bibliographie Travail et condition féminine publiée en

\footnotetext{
Sur le BIEF voir Thébaud 2004 : 163-184.

Daune-Richard \& Devreux 1985 : 39-53.

Les dernières en date en français Agone 2010 et Dorlin 2009.

4 Entre autres, Dictionnaire historique du féminisme 2007 [2000] ; Clio HFS 2004.
} 
1977 par Madeleine Guilbert, Nicole Löwit et Marie-Hélène Zylberberg-Hocquart - instrument de travail indispensable selon Michelle $\operatorname{Perrot}^{5}$ - a représenté pendant longtemps à mes yeux la borne ultime de l'usage du syntagme condition féminine et je l'avais même qualifiée ailleurs de «butte-témoin». L'intérêt porté aujourd'hui à une autre échelle, celle de la world history, et l'utilisation des possibilités documentaires ouvertes par les portails consultables par internet (Persée, Revues.org, Cairn pour la France; Erudit au Canada ; JStor ou Muse aux États-Unis...) m’ont conduit à réviser cette affirmation et à identifier d'autre usages en d'autres lieux, y compris dans l'immédiat contemporain. Les rapports demandés par les organisations internationales - l'ONU ou l'UNESCO - sur les pays du Sud ou pays émergents, emploient systématiquement l'expression «condition féminine» pour désigner la situation spécifique et défavorisée des femmes dans ces pays. Dans le même type de rapports internationaux, le terme genre dans le syntagme "genre du développement » est utilisé dans un sens similaire à celui de condition matérielle des femmes, et non dans le sens théorique défini par Joan Scott en 1986. Je vais m'efforcer de dresser ici un bilan nécessairement succinct dans le cadre imparti à cet article - et qui pourra paraître schématique - des usages de ces différents termes, tout en précisant qu'il s'agit d'un regard occidental et français (même si l'on peut constater certains points communs avec les Québécois-e-s).

\section{Parcours disciplinaires de la condition féminine aux rapports sociaux de sexe}

Le premier élément à souligner est la différence d'intégration de ces concepts selon les disciplines. Dans la sociologie française l'on emploie, successivement, mais aussi de façon concomitante, toutes ces catégories à partir de la rupture épistémologique introduite par les féministes des années 1968, en particulier par Christine Delphy qui a avancé les notions de patriarcat, d'oppression et de classe de sexe ${ }^{6}$. Dans la discipline historique, plus frileuse en France sur la conceptualisation, on est passé de la condition féminine - celle des

\footnotetext{
5 Perrot 1978.

6 Delphy 1970.
} 
ouvrières ayant été privilégié - au genre ${ }^{7}$ tardivement toléré en histoire (2002) à partir du colloque de l'université de Rennes ${ }^{8}$, avec cependant des étapes par la différence des sexes (ou entre les sexes ${ }^{9}$ ) ou encore le masculin/féminini ${ }^{10}$.

Depuis les philosophes de l'Antiquité, Aristote et Platon, de nombreux discours existent sur la nature et la hiérarchie entre les sexes. Mais, après la Seconde Guerre mondiale, l'importance prise par le marxisme dans les sciences sociales françaises explique le succès du terme condition féminine, toujours rapporté au travail, et décalqué de l'expression condition ouvrière ${ }^{11}$. En effet, depuis L'Origine de la famille, de la propriété privée et de l'État de Friedrich Engels (1884), l'assujettissement des femmes et la division sexuelle du travail, en lien avec la société capitaliste, ont été affirmés, même si la domination masculine a existé bien avant la formation des classes sociales et de l'État, comme l'ont souligné les anthropologues ${ }^{12}$. En auscultant en 1947 les changements de la condition féminine, le sociologue Pierre Fougeyrollas est l'un des premiers à prendre en compte les pouvoirs dans la famille grâce à une enquête par questionnaire publiée dans la revue Population sur «la prédominance du mari ou de la femme dans le ménage $»^{13}$. La même revue rend compte en 1957 de la condition ouvrière féminine et du travail des femmes en citant une enquête de Michèle Aumont (qui s'est établie en usine) suggérant - en des termes qui signalent à la fois la modernité et une conception traditionnelle de l'épouse-mère - de «déprolétariser la condition ouvrière, et de revaloriser celle de la ménagère. L'usine et le foyer doivent assurer la

7 Scott 1988 (traduction de l'article paru en anglais en 1986 dans les Cabiers du Grif intitulé justement «Le genre de l'histoire ») : 125-153.

8 Publié l'année suivante, voir Capdevila et al. 2003.

9 Michelle Perrot l'emploie dès 1984 dans Une histoire des femmes est-elle possible? Il est présent dans l'ouverture du premier numéro de Clio. Histoire, Femmes et Sociétés en 1995.

10 Sohn \& Thélamon 1998.

11 Journées d'étude du travail de la femme des 31 janvier et 1er février 1947, Population, 1947, 1 : 206-207 (à noter le singulier que je souligne, voir la même remarque pour la note 15$)$.

12 Mathieu 1971 ; Tabet 1979 ; Héritier 1996.

13 Population, 1951, 1 : 83-102. 
même dignité, car la femme n'est pas une machine, la femme n'est pas une marchandise... $»^{14}$. En 1964, Andrée Michel et Geneviève Texier dénoncent dans $\mathrm{La}$ condition de la Française d'aujourd'bui la «femme-objet soumise ou sublimée existant par et pour un homme $»^{15}$. La revue Population poursuit son étude des modes de vie par celle des mariages, des naissances et des divorces rejoignant ainsi la démographie historique qui reconstitue les familles, mais ne voit pas toujours les femmes ${ }^{16}$. Sous la plume d'Évelyne Sullerot, la prestigieuse revue d'histoire, Annales ESC, aborde en 1967 la notion de condition féminine, par le biais d'un compte rendu d'un petit ouvrage de J.E. Havel, prétexte à la sociologue pour décrire les aspects contemporains de la condition féminine ${ }^{17}$.

Il est donc logique que ce soit à partir du début de la délégitimation du marxisme (marqué en France par le succès de L'Archipel du Goulag de Soljenitsyne traduit en 1974) que l'expression condition féminine soit fortement remise en cause, même si elle perdure en particulier dans les institutions ministérielles ou partidaires. En 1974, Valéry Giscard d'Estaing, élu président de la République, innove avec un secrétariat d'État à la Condition féminine confié à Françoise Giroud, tandis que le parti socialiste crée dans sa direction, la même année, un poste de secrétaire à la Condition féminine. En 1986, avec la première cohabitation, le ministère des Droits de LA femme (attribué en 1981 par François Mitterrand à Yvette Roudy) cède la place à une Délégation à la Condition féminine rattachée au ministère des Affaires sociales, signe d'un retour en arrière à la fois institutionnel et sémantique sur la question des droits des femmes.

Dans les sciences sociales (et particulièrement en sociologie), l'abandon de l'approche en termes de condition féminine se fit par un déplacement du regard et une nouvelle réflexion théorique sur le sujet, inspirés par la théorie féministe ${ }^{18}$. Le terme de rapports sociaux

\footnotetext{
14 Compte-rendu dans Populations, 12/2, $1957: 364$.

15 Michel \& Texier 1964.

16 Guelaud-Leridon 1966 : 275-332; Thébaud 2009.

17 Annales ESC, 3, $1967:$ 639-641.

18 Ferrand 1993 : 5-6.
} 
de sexe s'impose alors porté en particulier par Danièle Kergoat ${ }^{19}$ et les membres de l'APRE (Atelier production/reproduction) ${ }^{20}$ et du CLEF (Centre lyonnais d'études féministes) qui publie en 1984 les actes d'un colloque sur Le Sexe du travail ${ }^{1}$. Si le terme rapports sociaux de sexe partage avec condition féminine le même socle théorique - le marxisme - ce premier terme souligne des relations de pouvoir des hommes sur les femmes. La condition féminine de son côté, en renvoyant aux caractéristiques biologiques et en particulier à la maternité, énonce la complémentarité et la spécificité féminine. L'expression "rôles sociaux sexués » quant à elle a été critiquée parce que relevant de la même assignation au destin naturel de l'espèce ${ }^{22}$. Dans l'expression rapports sociaux de sexe, le présupposé est qu'il s'agit de construits sociaux créés par l'existence de deux groupes sociaux spécifiques, les hommes et les femmes. La base matérielle de ces rapports sociaux de sexe est le travail, marqué par une division sexuelle, variable selon les lieux et les moments. Cette théorie critique a redéfini certains concepts clés (dont l'objet travail avec l'articulation production et reproduction), déplacé les frontières intradisciplinaires de la sociologie du travail et de la sociologie de la famille, en même temps qu'elle a élargi les débats sur des thèmes sociologiques fondamentaux: reproduction sociale et changement, structures et acteurs/actrices, pratiques sociales et représentations ${ }^{23}$. Dans la discipline historique s'est développée une histoire des rapports entre syndicalisme et féminisme et une histoire ouvrière du travail féminin ${ }^{24}$. Dès 1978, Joan Scott et Louise Tilly faisaient le lien entre famille et travail dans une histoire comparée des ouvrières de la France et de l'Angleterre ${ }^{25}$. Il faut attendre 1986 pour que soit posée la question des pouvoirs en dehors de la sphère du travail ${ }^{26}$.

\footnotetext{
19 Kergoat 1982, 1992.

20 Cabiers de l'APRE à partir de 1984.

21 Collectif 1984.

22 Kergoat 2007 : 35-44.

23 Daune-Richard \& Devreux 1992 : 7-30 ; Laufer et al. 2003.

24 Thébaud 1998.

25 Scott \& Tilly 1987 [1978].

26 Dauphin et al. 1986 ; sur le bilan historiographique de l'Histoire des femmes voir Thébaud 2007 [1998].
} 


\section{De l'affirmation du genre à sa déconstruction}

1986 est l'année où Joan Scott publie aux États-Unis un article sur le genre devenu une référence/révérence obligée ; c'est sa traduction notoirement rapide en français (1988) et sa conceptualisation qui lui ont donné un grand retentissement. Joan Scott énonce ses outils, la théorie de Michel Foucault (avec sa " politique de la sexualité ») et la psychanalyse (Freud revisité par Lacan), pour prendre en compte le processus d'identité du sujet. C'est à travers le langage qu'est construite l'identité sexuée. Le genre est un champ par le moyen duquel le pouvoir est articulé. Joan Scott se réfère au poststructuralisme qui définit les catégories historiques d'analyse comme contextualisées et situées, résultant des processus linguistiques. Au lieu de considérer la catégorie femme comme préexistante, elle analyse la manière dont les sujets sont constitués comme différents, et pas forcément par des catégories binaires. On est donc passé de la référence marxiste des rapports sociaux de sexe, catégorie trop descriptive selon Scott, à un système de genre qui a une existence propre et qui s'appuie sur le post-structuralisme et la French Theory (Lacan, Foucault, Barthes, Derrida).

En 1999, s'élevant contre un usage devenu selon elle banalisé et routinier du mot genre qui lui fait perdre son caractère critique, et dénonçant le retour en force d'une pensée biologisante, tout comme les effets pervers d'une division entre le sexe biologique qui accrédite son anhistoricité et le sexe social, de même que la bicatégorisation, Joan Scott a remis en question l'emploi du concept de genre (voir l'article traduit dans ce même numéro). Il faut sans doute voir aussi dans cette démarche une influence indirecte de la théorie queer qui se développe aux États-Unis à partir de 199027. Elle aborde le rivage français (il en est de même au Québec) tardivement (Butler est traduite à partir de 2005); mais elle a contribué indirectement à développer, en France, une sociologie et une histoire des sexualités à partir de la mise en cause des normes hétérosexuelles et de la mise en valeur des pratiques transgenre, de l'inversion, ou du travestissement ${ }^{28}$. C'est la division hiérarchique des humains en deux

\footnotetext{
27 Butler 2005 [1990].

28 Steinberg 2000.
} 
genres qui construit la différence sexuelle - le genre précède le sexe et Butler avance que, dans ce paradigme, le sexe et le genre, constructions culturelles, sociales et politiques, sont susceptibles d'être transformés. Le genre n'est pas inné, mais il se réalise à travers les normes et les injonctions quotidiennes : Butler dit ainsi qu'il a un caractère performatif, que c'est une "dimension du discours qui a la capacité de produire ce qu'il nomme ", et qu'aucune identité n'est définie a priori. Elle affirme que tout se construit par le langage et le symbolique. Il s'agit donc d'une remise en cause profonde de l'essentialisme et de la naturalisation, y compris celle des corps. La philosophe remet en cause également la catégorie femme/s qui uniformise et naturalise des expériences sociales très diverses au détriment de la diversité des positions et questionne ainsi le féminisme. L'accent mis sur les discours et les pouvoirs dans le sillage de Foucault et de Lacan peut faire prendre des distances avec les réalités concrètes, celles de l'oppression et de la domination qui peuvent concerner tout autant la classe, la race, l'âge, etc. ${ }^{29}$ et avec les structures sociales inégalitaires et hiérarchiques. C'est aussi une autre des raisons - outre les traductions tardives - qui expliquent le retard ou les réticences de la réception de la théorie queer en France très marquée par les approches matérialistes ${ }^{30}$.

Avant les sciences politiques qui ont découvert très récemment le genre et qui n'ont pas capitalisé les études antérieures des autres disciplines, l'histoire en France - plus tardivement cependant que la sociologie - a intégré le concept, même si les premières thèses d'histoire incluant une étude de genre (sans parfois le nommer) ont été soutenues en 1987-198831. Dès 1987, Eleni Varikas dans sa thèse sur les femmes en Grèce au XIXe siècle - malheureusement non publiée en français - forge le concept de «conscience de genre $»^{32}$. C’est elle aussi qui, avec Christine Planté et Michèle Riot-Sarcey, a

29 L'articulation complexe des catégories ou des appartenances est aujourd'hui définie par le terme d'intersectionnalité.

$30 \quad$ Vidal 2006 : 229-239; Baril 2007 ; Agone 2010.

31 Chenut 2005 [1988]. Dubesset \& Zancarini-Fournel 1993 [1988] ;

32 Varikas 1987. 
édité le numéro des Cabiers du Grif intitulé «le genre de l'histoire » avec, entre autres, l'article de Joan Scott cité ci-dessus.

Dès 1995, le MAGE (Groupe de recherche du CNRS sur marché du travail et genre) imposait progressivement l'emploi du mot genre en sociologie, en publiant dans un premier temps un bulletin puis la revue Travail, genre et sociétés. Journées d'études, colloques et ouvrages ont banalisé le terme ${ }^{33}$. Les grands éditeurs l'ont accepté en 2000 avec la publication de Genre et politique, chez Gallimard. La même année, Clio. Histoire, Femmes et Sociétés l'emploie explicitement dans un titre "Le genre de la nation ", même si le titre du n 10 avait déjà joué avec le mot en évoquant le «mauvais genre» des femmes travesties ${ }^{34}$. Le premier ouvrage d'histoire, issu d'un colloque, incluant le mot genre dans son titre, date de $2003^{35}$. Le terme vient tout juste de conquérir sa légitimité historienne quand la Commission générale de terminologie et de néologie en récuse l'emploi dans les sciences sociales, en souhaitant qu'il soit réservé à la littérature et à la grammaire ${ }^{36}$.

Cependant le terme genre a intégré la sphère politique européenne (et internationale) avec le gender mainstreaming, c'est-à-dire la prise en compte des effets de genre dans les politiques publiques : il s'agit d'une démarche intégrée de l'égalité des chances venue de l'Europe $\mathrm{du}$ nord-ouest avec un autre socle théorique. Les paradoxes de l'universalisme français ${ }^{37}$ sont abandonnés au profit de la théorie de Rawls, mais «l'égalité des chances » est un autre concept et une autre histoire... à suivre.

\footnotetext{
33 Maruani 2005.

34 Auslander \& Zancarini-Fournel 2000 ; Bard \& Pellegrin 1999.

35 Capdevila et al. 2003.

36 Journal officiel de la République française, 169, 22 juillet 2005.

37 Scott 1997.
} 


\section{Bibliographie}

La bibliographie sur le genre est immense en particulier dans le monde anglophone. Seuls les livres et articles cités dans l'article sont répertoriés dans cette bibliographie qui est donc loin d'être exhaustive.

Agone, 2010, « Comment le genre trouble la classe », 43.

Auslander Leora \& Michelle ZANCARINi-Fournel (dir.), 2000, «Le genre de la nation », Clio. Histoire, Femmes et Sociétés, 12.

BARIL Audrey, 2007, «De la construction du genre à la construction du "sexe": les thèses féministes postmodernes dans l'œuvre de Judith Butler", Recherches féministes, 20, 2, p. 61-90.

Bulletin d'Information des études féminines (BIEF), 1985, «Sexes et catégories », Université de Provence, 17.

ButLer Judith, 2004 [1 19e éd. 1996], Le ponvoir des mots. Politique du performatif, Paris, Éditions Amsterdam.

Butler Judith, 2005 [1 éd. 1990], Trouble dans le genre. Pour un féminisme de la subversion, Paris, La Découverte.

ButLer Judith, 2006 [1re éd. 2004], Défaire le genre, Paris, Éditions Amsterdam.

Capdevila Luc, Cassagne Sophie, Cocaud Martine et al. (dir.), 2003, Le genre face aux mutations. Masculin et féminin, du Moyen Age à nos jours, Rennes, Presses universitaires de Rennes.

Chenut Helen Harden, 2005, The Fabric of Gender: Working-Class Culture in Third Republic France, University Park, Pennsylvania State University Press [thèse de doctorat Université de Paris 7 (1988), publiée aux États-Unis dans une version profondément remaniée].

Clio. Histoire, Femmes et Sociétés, 2004, Les Mots de l'bistoire des femmes, Toulouse, Presses universitaires du Mirail.

Collectif, 1984, Le Sexe du travail. Structures familiales et système productif, Grenoble, Presses universitaires de Grenoble.

Daune-Richard Anne-Marie \& Anne-Marie Devreux, 1985, «La construction sociale des catégories de sexe », BIEF, p. 39-53.

Daune-Richard Anne-Marie \&Anne-Marie Devreux, 1992, «Rapports sociaux de sexe et conceptualisation sociologique ", Recherches féministes, 5, 2, p. 7-30.

DAuphin Cécile et al., 1986, "Culture et pouvoir des femmes: essai d'historiographie ", Annales ESC, 2, p. 271-293. 
DeLPHY Christine, 1970, «L'ennemi principal », Partisans, «Libération des femmes année zéro », p. 54-55.

DoruIN Elsa (dir.), 2009, Sexe, race, classe. Pour une épistémologie de la domination, Paris, Presses universitaires de France.

FERRAND Michèle, 1993, «Éditorial », Sociétés contemporaines, 16, 1, p. 5-6.

Dubesset Mathilde \& Michelle Zancarini-Fournel, 1993, Parcours de femmes. Réalités et représentations. Saint-Etienne 1880-1950, Lyon, Presses universitaires de Lyon, coll. «Centre Pierre Léon » [issu de la thèse de doctorat, soutenue en 1988 à l'Université de Lyon 2].

GUELAUd-LERIDON Françoise, 1966, « Recherches sur la condition féminine dans la société d'aujourd'hui », Population, 2, p. 275-332.

Kergoat Danièle, 1982, Les Ouvrières, Paris, le Sycomore.

—, 1992, «À propos des rapports sociaux de sexe », Revue M, 53-54, p. 16-20.

—, 2007, «Division sexuelle du travail et rapports sociaux de sexe», in Helena Hirata et al. (dir.), Dictionnaire critique du féminisme, Presses universitaires de France, p. 35-44.

LAUfer Jacqueline, MARRY Catherine \& Margaret MARUANi (dir.), 2003, Le travail du genre. Les sciences sociales du travail à l'épreuve des différences de sexe, Paris, La Découverte.

Maruani Margaret (dir.), 2005, Femmes, genre et sociétés. L'état des savoirs, Paris, La Découverte.

Michel Andrée \& Geneviève Texier, 1964, La condition de la Française d'aujourd'bui, Paris, Gonthier, 2 tomes, 246 p. et 243 p.

Perrot Michelle (dir.), 1978, «Travaux de femmes », Le Mowvement social, 105.

—, 1984, Une Histoire des femmes est-elle possible?, Marseille/Paris, Rivages.

SCOTT Joan W., 1988 [1986], "Genre : une catégorie utile d'analyse historique », Cabiers du Grif, « Le genre de l'histoire », 37/38, p. 125-153.

—, 1998 [1996], La citoyenne paradoxale: les féministes françaises et les droits de l'homme, Paris, Albin Michel.

ScOTT Joan W. \& Louise TiLly, 1987 [1978], Les femmes, le travail et la famille, Marseille/Paris, Rivages.

SOHN Anne-Marie \& Françoise THÉLAMON (dir.), 1998, Une bistoire sans les femmes estelle possible ?, Paris, Perrin.

STEINBERG Sylvie, 2000, La confusion des sexes. Le travestissement de la Renaissance à la Révolution, Paris, Fayard. 
THÉBAud Françoise, 2004, «Femmes, féminisme, recherches : regard sur le Bief», in Marcel Bernos \& Michèle BITTON (dir.), Femmes, filiations, familles. Mélanges en l'honneur d'Yvonne Knibiebler, Aix-en-Provence, Presses universitaires de l'université de Provence, p. 163-184.

—, 2007 (1998), Écrire l'histoire des femmes et du genre, Lyon, ENS Éditions.

—, 2009, «Histoire des femmes et du genre et démographie historique: convergences, malentendus, divergences ", in Michel ORIS, Guy BRUNET et al. (dir.), Une démographie au féminin - A Female Demography, Peter Lang.

VARIKAS Eleni, 1987, La Révolte des Dames: Genèse d'une conscience féministe en Grèce, Athènes, Éditions Archives Historiques, Banque Commerciale de Grèce [version éditée en grec, édition Katarti, 1996, issue de la thèse, en français, soutenue à l’Université Paris 7 en 1987].

—, 2006, Penser le sexe et le genre, Paris, Presses universitaires de France,.

VIDAL Jérôme, 2006, «À propos du féminisme. Judith Butler en France : trouble dans la réception », Mouvements, 47/48, septembre/décembre, p. 229-239. 